\title{
Numerical Simulations of Combined Effects of Terrain Orography and Thermal Stratification on Pollutant Distribution in a Town Valley
}

\author{
S. Kenjerešs, K. Hanjalić, and G. Krstovi ć \\ Department of Applied Physics, Delft University of Technology \\ Lorentzweg 1, 2628 CJ Delft, The Netherlands \\ kenjeres@ws.tn.tudelft.nl; hanjalic@ws.tn.tudelft.nl, \\ WWW home page: http://www.ws.tn.tudelft.nl
}

\begin{abstract}
Combined effects of terrain orography and thermal stratification on the dispersion of pollutants in a mountainous town valley over a diurnal cycle are numerically simulated by a time-dependent Reynoldsaveraged Na vier-Stok es (T-RANS) approdac The T-RANS model was incorporated in to a finite $\mathrm{v}$ olume NS soler for three-dimensional nonorthogonal domains, using Cartesian vector and tensor components and collocated variable arrangement. Prior to the full scale simulations, the T-RANS approach was validated in test situations where the effects of thermal stratification and terrain orography are separated, showing good agreement with the available experimental and simulation data. The full scale simulations were performed in a realistic orography over two diurnal cycles for tw ocases of the initial thermal stratification, both with a prescribed time and space variation of groundemperature and pollutan t emission - reflecting the daily activities in the town. The results confirmed that T-RANS approach can serve as a powerful tool for predicting local environments at micro and meso scales.
\end{abstract}

\section{Introduction}

Most urban areas are contin uous sources of heat and pollution as a result of a variety of human activities, e.g. industrial processes, transportation, agriculture, etc. In addition, a high percentage of urban areas is covered with concrete and asphalt which store and reflect incoming radiation causing a significantly w armersurface-layer air than that of their natural surroundings. As a result, the urban areas form a kind of local heat islands in the surrounding countryside, Stull [9]. Urban city landscape with tall buildings and streets of different sizes together with surrounding terrain orography create very complex local geometry. These local boundary conditions together with an imposed initial temperature distribution in atmosphere (thermal stratification) form a complex interactions mechanism betw een heat transfer and corresponding atmospheric pollutant emission. The prediction of this complex interaction mechanism is of vital importance for estimating possible toxic pollutant distribution that may pose a risk to human health. It is also the major prerequisite for optimum control of air quality:

P.M.A. Sloot et al. (Eds.): ICCS 2002, LNCS 2329, pp. 266-275, 2002.

(C) Springer-Verlag Berlin Heidelberg 2002 
future city planning and optimum location of industrial zones, design of city transportation system, control of traffic and industrial activities during critical meteorological periods, etc. Current practice relies on semi-empirical methods and simple integral modelling of pollutant dispersion with prescribed wind conditions, whereas situations at micro and meso scales dominated by buoyancy are usually beyond the reach of such models. Large eddy simulations (LES) is a possible option, but a hybrid LES/RANS approach ('ultra' CFD problems; Hunt [2]) seems a more viable option that can provide, under lower costs, required detailed insights into above mentioned complex phenomena.

In this paper, we propose the transient Reynolds-averaged Navier-Stokes (TRANS) approach as potentially efficient, numerically robust and physically accurate method for simulation of combined effects of terrain orography and thermal stratification on pollutant dispersion. These effects will be analysed by performing simulations of a realistic environmental problem of a medium-size valley town with significant residential and industrial pollution. The critical periods are the winter cloudy windless days when the lower atmosphere in the valley is capped with an inversion layer preventing any convection through it. The air movement and the pollutant dispersion are solely generated by the day ground heating in surroundings and urban areas. While any realistic conditions can be imposed, we consider at present - as a part of a preliminary study - an idealised situation with space and time sinusoidal variation of temperature and concentration, both imitating two diurnal cycles. In order to accommodate a very complex terrain orography, a finite-volume Navier-Stokes solver for three-dimensional flows in structured non-orthogonal geometries, based on Cartesian vector and tensorial components and collocated variable arrangement, was applied.

Prior to the full scale simulations that include both effects - thermal stratification and terrain orography - the T-RANS approach was validated in cases of unsteady turbulent penetrative convection of unstable mixed layer and classical Rayleigh-Bénard convection over flat and wavy surfaces of different topology and over a range of $R a$ numbers.

\section{The Time-Dependent RANS (T-RANS): equations and subscale models}

This approach can be regarded as Very Large Eddy Simulations (VLES) in which the stochastic motion is modelled using a $\langle k\rangle-\langle\varepsilon\rangle-\left\langle\overline{\theta^{2}}\right\rangle$ Algebraic Stress/Flux/Concentration (ASM/AFM/ACM) single-point closure models as the "subscale model", where \langle\rangle denoted the time-resolved motion. The turbulent stress tensor, $\tau_{i j}=\left\langle u_{i} u_{j}\right\rangle$, heat flux vector, $\tau_{\theta i}=\left\langle\theta u_{i}\right\rangle$ and concentration flux vector, $\tau_{c i}=\left\langle c u_{i}\right\rangle$, were derived by truncation of the modelled RANS parent differential transport equations by assuming weak equilibrium, i.e. $(D / D t-\mathcal{D}) \overline{\phi u_{i}}=0$, but retaining all major flux production terms (all treated as time-dependent). In contrast to Large Eddy Simulation (LES), the contribution of both modes to the turbulent fluctuations are of the same order of magnitude. 
Environmental fluid flows are described by standard conservation laws for mass, momentum, energy and concentration. For the resolves ('filtered') motion, equations can be written in the essentially same form as for the LES:

$$
\begin{array}{r}
\frac{\partial\left\langle U_{i}\right\rangle}{\partial t}+\left\langle U_{j}\right\rangle \frac{\partial\left\langle U_{i}\right\rangle}{\partial x_{j}}=\frac{\partial}{\partial x_{j}}\left(\nu \frac{\partial\left\langle U_{i}\right\rangle}{\partial x_{j}}-\tau_{i j}\right) \\
-\frac{1}{\rho} \frac{\left(\langle P\rangle-P_{r e f}\right)}{\partial x_{i}}+\beta g_{i}\left(\langle T\rangle-T_{r e f}\right) \\
\frac{\partial\langle T\rangle}{\partial t}+\left\langle U_{j}\right\rangle \frac{\partial\langle T\rangle}{\partial x_{j}}=\frac{\partial}{\partial x_{j}}\left(\frac{\nu}{\operatorname{Pr}} \frac{\partial\langle T\rangle}{\partial x_{j}}-\tau_{\theta j}\right) \\
\frac{\partial\langle C\rangle}{\partial t}+\left\langle U_{j}\right\rangle \frac{\partial\langle C\rangle}{\partial x_{j}}=\frac{\partial}{\partial x_{j}}\left(\frac{\nu}{S c} \frac{\partial\langle C\rangle}{\partial x_{j}}-\tau_{c j}\right)
\end{array}
$$

where \langle\rangle stands for resolved ensemble-averaged quantities and $\tau_{i j}, \tau_{\theta j}$ and $\tau_{c j}$ represent contributions due to unresolved scales to momentum, temperature and concentration equation respectively, which were provided by the subscale model. In the present work which is still at the preliminary stage, the adopted 'subscale' expression are given as follows. For turbulent stresses we applied eddy viscosity expression:

$$
\tau_{i j}=-\nu_{t}\left(\frac{\partial\left\langle U_{i}\right\rangle}{\partial x_{j}}+\frac{\partial\left\langle U_{j}\right\rangle}{\partial x_{i}}\right)+\frac{2}{3}\langle k\rangle \delta_{i j}
$$

The turbulence heat and concentration fluxes are expressed by AFM counterparts:

$$
\begin{gathered}
\tau_{\theta i}=-C_{\phi} \frac{\langle k\rangle}{\langle\varepsilon\rangle}\left[\tau_{i j} \frac{\partial\langle T\rangle}{\partial x_{j}}+\xi \tau_{\theta j} \frac{\partial\left\langle U_{i}\right\rangle}{\partial x_{j}}+\eta \beta g_{i}\left\langle\theta^{2}\right\rangle\right] \\
\tau_{c i}=-C_{\phi} \frac{\langle k\rangle}{\langle\varepsilon\rangle}\left[\tau_{i j} \frac{\partial\langle C\rangle}{\partial x_{j}}+\xi \tau_{c j} \frac{\partial\left\langle U_{i}\right\rangle}{\partial x_{j}}\right]
\end{gathered}
$$

The closure of the expressions for subscale quantities is achieved by solving the equations for turbulence kinetic energy $\langle k\rangle$, its dissipation rate $\langle\varepsilon\rangle$ and temperature variance $\left\langle\theta^{2}\right\rangle$, resulting in three-equation model $\langle k\rangle-\langle\varepsilon\rangle-\left\langle\theta^{2}\right\rangle$, Kenjereš and Hanjalić [4]:

$$
\begin{array}{r}
\frac{D\langle k\rangle}{D t}=\mathcal{D}_{k}+P_{k}+G_{k}-\langle\varepsilon\rangle \\
\frac{D\langle\varepsilon\rangle}{D t}=\mathcal{D}_{\varepsilon}+P_{\varepsilon 1}+P_{\varepsilon 2}+G_{\varepsilon}-Y \\
\frac{D\left\langle\theta^{2}\right\rangle}{D t}=\mathcal{D}_{\theta}+P_{\theta}-\left\langle\varepsilon_{\theta}\right\rangle
\end{array}
$$

\section{Results}

\subsection{Unsteady turbulent penetrative convection of unstable mixed layer}

The ability of the proposed Algebraic-Stress-Flux-Concentration subscale Model (ASM/AFM/ACM) to reproduce correctly the flows with a strong thermal strat- 

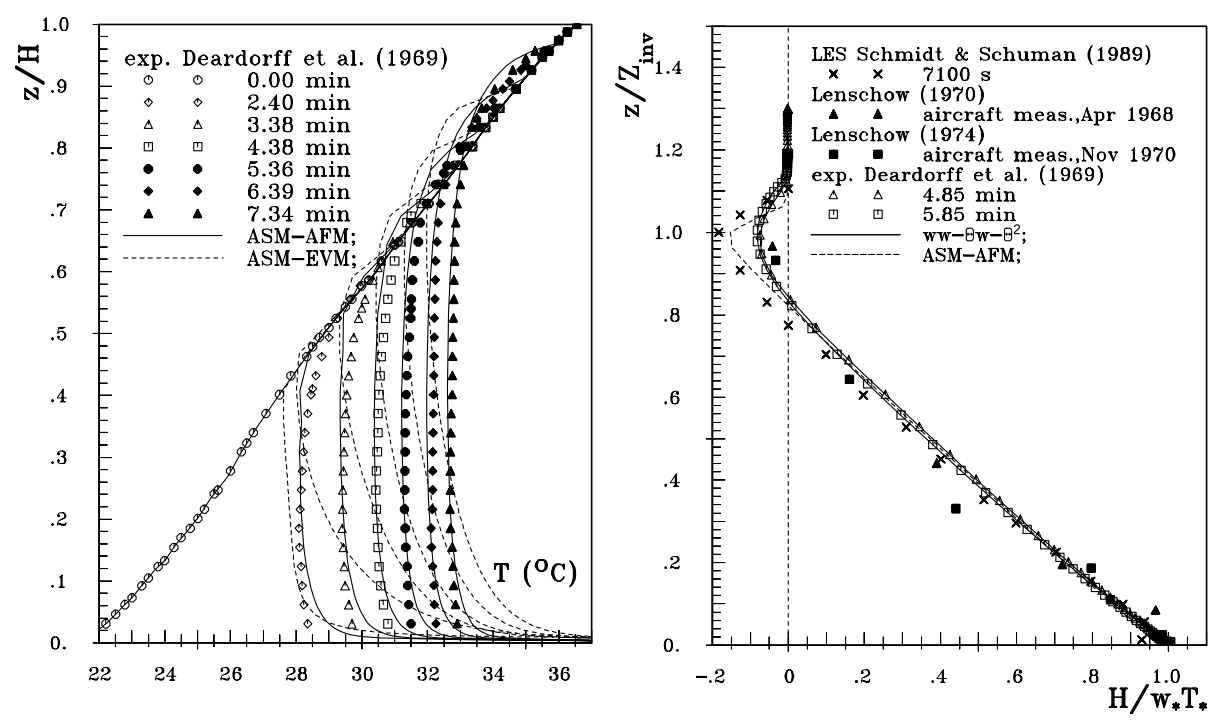

Fig. 1. Time evolution of vertical temperature profiles in a mixed layer heated from below -left, Normalised vertical heat flux in a mixed layer heated from below- right: comparison between experiments and simulations.

ification was first tested on unsteady turbulent penetrative convection of an unstable mixed layer. Due to heating from bellow, the initially stable environment in the near-ground region becomes unstable and interactions between stable and unstable regions occur. With further intensification of the heat transfer in the vertical direction, the location of the interface between the two regions moves up with time, causing the mixed turbulent ground layer to grow into a stable region. Another feature of the phenomenon is the entrainment of overlaying nonturbulent fluid into mixed layer causing very steep gradients at the interface as well as a reversal of the sign of the heat flux as a result of the compensating cooling of the fluid in the stable region, Nieuwstadt et al. [7]. These features explain why turbulent penetrative convection represents a very challenging test case for turbulence models, Mahrt [6]. Fig. 1a shows the time evolution of temperature profiles obtained with ASM/AFM model. The experimental data ( Deardorff et $a l$. .[1]; Willis and Deardorff [10]) are very well reproduced in the middle as well as at the upper edge of the mixed layer. A further simplification of turbulent heat flux to anisotropic eddy viscosity model (denoted with EVM), which basically excludes temperature variance from consideration, resulted in serious deterioration of the predicted results. This confirms that the AFM representation of turbulent heat flux is the minimum level of modelling which can provide close agreement with experimental temperature profiles. The predicted vertical heat flux suitably scaled with the inversion height and the product of the buoyancy velocity $\left(w_{*}=\left(\beta g Q_{s} Z_{i n v}\right)^{1 / 3}\right)$ and temperature $\left(T_{*}=Q_{s} / w_{*}\right)$ is shown in Fig. 1b. Here the solid line represents result obtained with the full second-order closure 

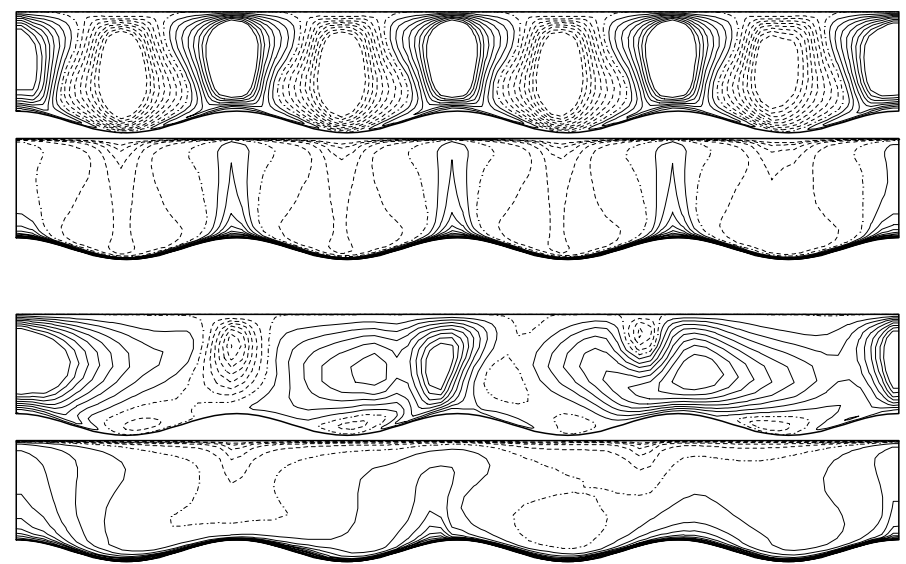

Fig. 2. Vertical velocity $\langle W\rangle$ and temperature $\langle T\rangle$ distributions in turbulent thermal convection over a terrain with $3 \mathrm{D}$ regular waviness, $S_{B}(x, y)=0.1 \cos (x \pi) \cos (y \pi)$, $R a=10^{7}, \operatorname{Pr}=0.71$; above- $\tau^{*}=50$, below- $\tau^{*}=200$.

for both momentum and temperature equations. As seen, the ASM/AFM result shows good agreement with several sets of laboratory and field measurements, and with LES data of Schmidt and Schumann [8].

\subsection{Transient Rayleigh-Bénard convection over horizontal wavy walls}

The potential of the T-RANS approach to capture the effects of bottom wall topology was verified against the DNS and LES results of Krettenauer and Schumann [5] for turbulent convection over wavy terrain - both under identical conditions. Their main finding was that the total heat transfer was only slightly affected by terrain orography compared to flat bottom wall situation. The gross feature of the flow statistics, such as profiles of turbulence fluxes and variance were not very sensitive to the variation of the bottom wall topology. On the other hand, they reported that the motion structure persisted considerably longer over the wavy terrain than over flat surfaces. While the Krettenauer and Schumann [5] were interested mainly in the final turbulence statistics under steady conditions, we extended our study to time evolution of turbulence quantities and large coherent structures. The effect of the horizontal wavy wall on the mean properties $\langle W, T\rangle$ is presented in Fig. 2. At the initial stage of heating, $\tau^{*}=\sqrt{\beta g \Delta T / H}=50$, all quantities show a regular flow pattern determined by the wall configuration. The plumes raise from the surface peaks and sink into the surface valleys, portraying 25 characteristic locations. At $\tau^{*}=200$ the initial organisation of the flow cannot be observed anymore. Thermal plumes occupy a significantly larger space and not only the regions close to the bottom surface peaks, as found in the initial phase of flow development, Hanjalić and Kenjereš [3]. It can be concluded that the T-RANS approach was able to capture impor- 


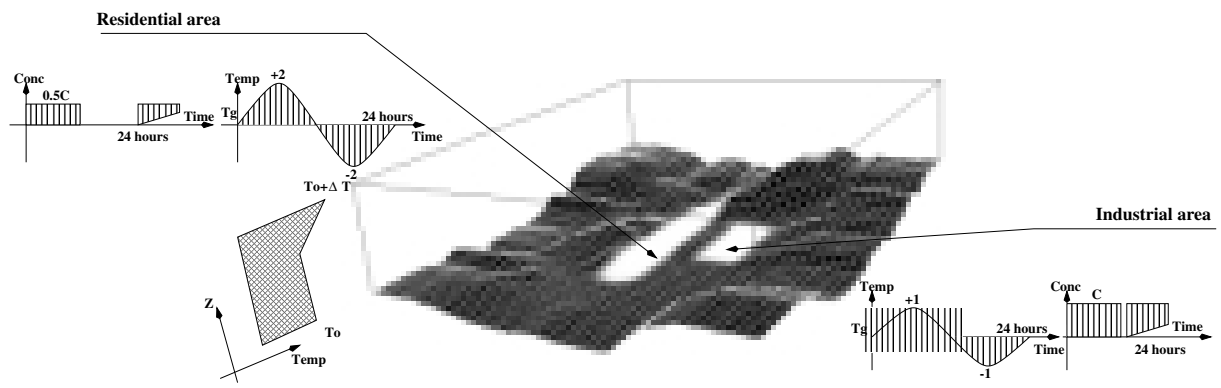

Fig. 3. Specification of imposed boundary and initial conditions for a middle-size town located in a mountain area; Two distinct areas (industrial and residential) are represented by different pollution emission $(C=100$ and $50 \%$ respectively) and different heat source intensities ( $T_{g}= \pm 1$ and \pm 2 respectively); Initial thermal stratification is also presented.

tant phenomena of time and spatial evolution of flow and turbulence structures which reflect specific horizontal surface topologies.

\subsection{Air circulation and pollutant dispersion in a town valley}

As an illustration of the potential of the T-RANS approach for predicting atmospheric thermal convection and pollutant transport in a real, full scale situation over a mountainous conurbation, we considered diurnal variation of air movement and pollutant dispersion over a medium-sized town situated in a mountain valley, with distinct residential and industrial zones. These two zones are represented by different pollution emission ( $C=50 \%$ and $100 \%$ respectively) and different heat source intensities $\left(T_{g}= \pm 2\right.$ and \pm 1 respectively) from surrounding areas, over two diurnal cycles, Fig. 3. Simulated domain covers an area of $12 \times 10 \times 2.5 \mathrm{~km}$, which was represented by an averaged mesh size of $100 \mathrm{~m}$ in each direction. Two consecutive diurnal cycles were simulated (0-24h, day (I) and day (II)) with a time step of $2.5 \mathrm{~min}$. Wall functions were applied for the ground plane. At the top boundary, we prescribed constant temperature and assumed symmetry boundary condition for the velocity. The side boundaries were artificially extended and treated as symmetries for all variables. Two different situation with respect to imposed thermal stratification were analysed. The imposed vertical profile of potential temperature of dry air is uniform in lower level and with identical linear distribution $(\Delta T=4)$ in upper level. The base of the inversion layer (the switch from uniform to linear temperature) is located at $z / H=2 / 3(\approx 1600 \mathrm{~m}$ from the valley deepest point $)$ for the first case and at $z / H=1 / 3(\approx 800 \mathrm{~m})$ for the second case. The domain height $(\mathrm{H})$ and the characteristic initial temperature gradients (or surface fluxes) give very high values of Rayleigh number, i.e. $\mathcal{O}\left(10^{17}\right)$.

Since the experimental measurements were not available for quantitative validation of presented simulations, no direct verification is possible. Instead, we 

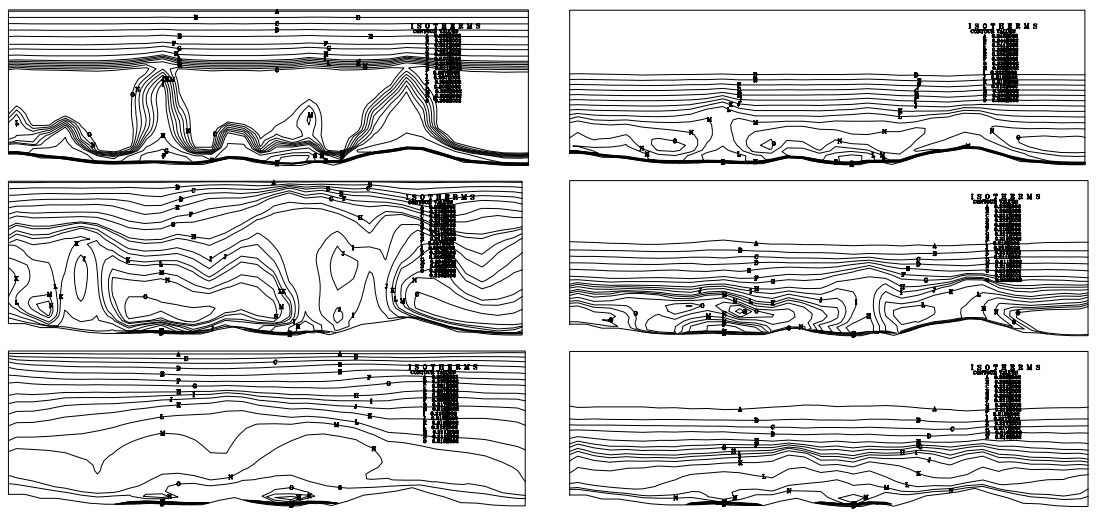

Fig. 4. Potential temperature distributions at characteristic location where both residential and industrial zone are present, for different time instants, $\tau=1$ p.m., 6 p.m. (day I), 10 a.m. (day II): left- weak stratification, right- strong stratification.

present some results that illustrate features of interest to environmental studies. The time evolution of the potential temperature distribution in a characteristic vertical plane that crosses both the residential and industrial zones, for two different stratifications, is shown in Fig. 4. In the initial stage of heating, strong thermal plumes appear, originating both from locally increased rate of heating (for industrial and residential zones) as well as from the hill orography. As time progresses, strong mixing in the lower layer is observed. At the beginning of the new cycle, due to nocturnal cooling, upper inversion layer is again moved towards the ground. As seen, stronger stratification causes significant damping of the thermal plume and transport activities, Fig. 5.
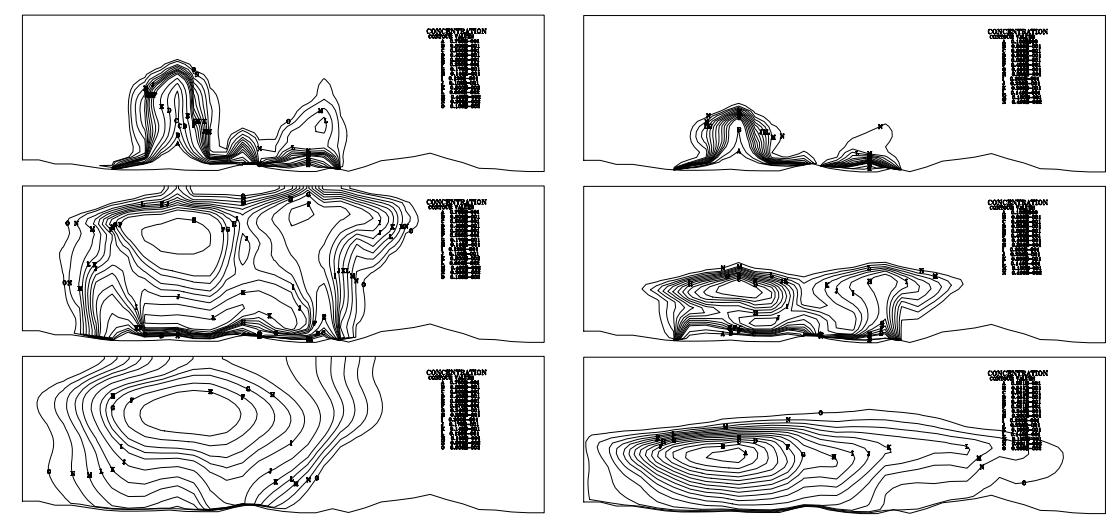

Fig. 5. Concentration distributions at characteristic location where both residential and industrial zone are present, for different time instants, $\tau=1$ p.m., 6 p.m. (day I), 10 a.m. (day II): left- weak stratification, right- strong stratification. 

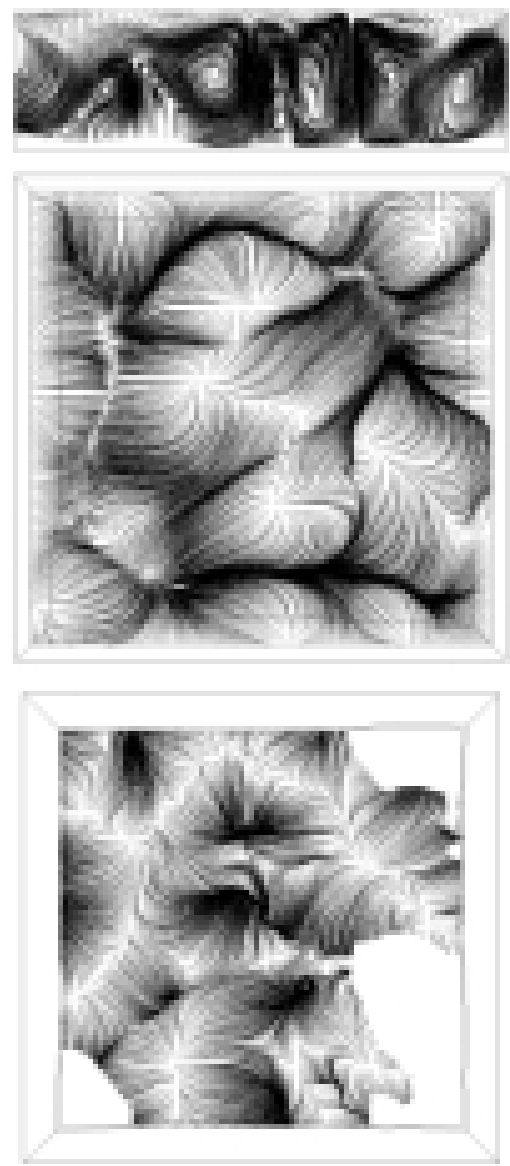
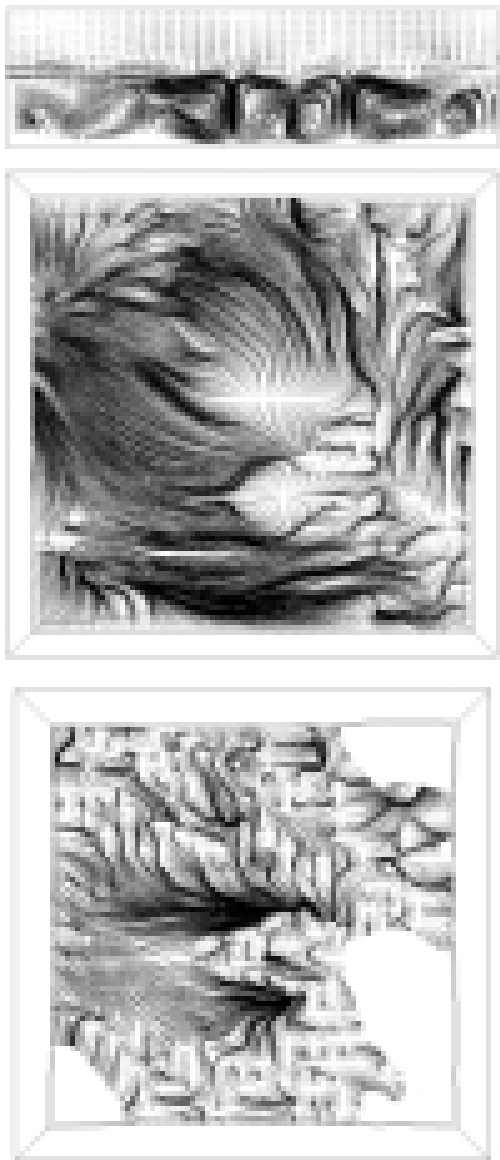

Fig. 6. Instantaneous trajectories in characteristic vertical plane (over residential and industrial zones) and two horizontal planes: first- at the base of inversion layer, second$50 \mathrm{~m}$ from ground; $\tau=6$ p.m., day II, case with weak stratification: left- weak stratification, right- strong stratification.

Same conclusion can be drawn from Fig. 6 where instantaneous trajectories in one vertical (residential+industrial zones) and two horizontal (at inversion layer location and at $50 \mathrm{~m}$ above the ground) planes. Since the capping inversion is closer to ground in the second case, vertical motion is significantly suppressed and the number of distinct thermal plumes is significantly larger in plane at $50 \mathrm{~m}$ from ground than for the weak stratification conditions. Numerical simulation also revealed interesting phenomena of up/down-slope inertial motions few hours after offset of heating/cooling of ground, as shown in Fig. 7. Finally, the three-dimensional concentration fields, represented by a characteristic concentration isosurface, are presented in Fig. 8. As expected, stronger stratification 

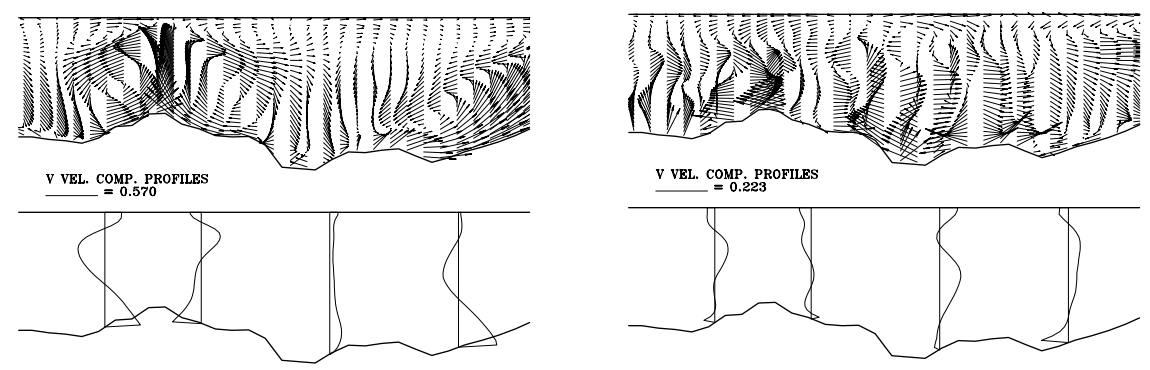

Fig. 7. Velocity vectors and horizontal velocity component profiles indicating an inertial motion, $2 \mathrm{hrs}$ after onset of heating/cooling, day (II), case with weak stratification: left- up-slope motion, right- down-slope motion.
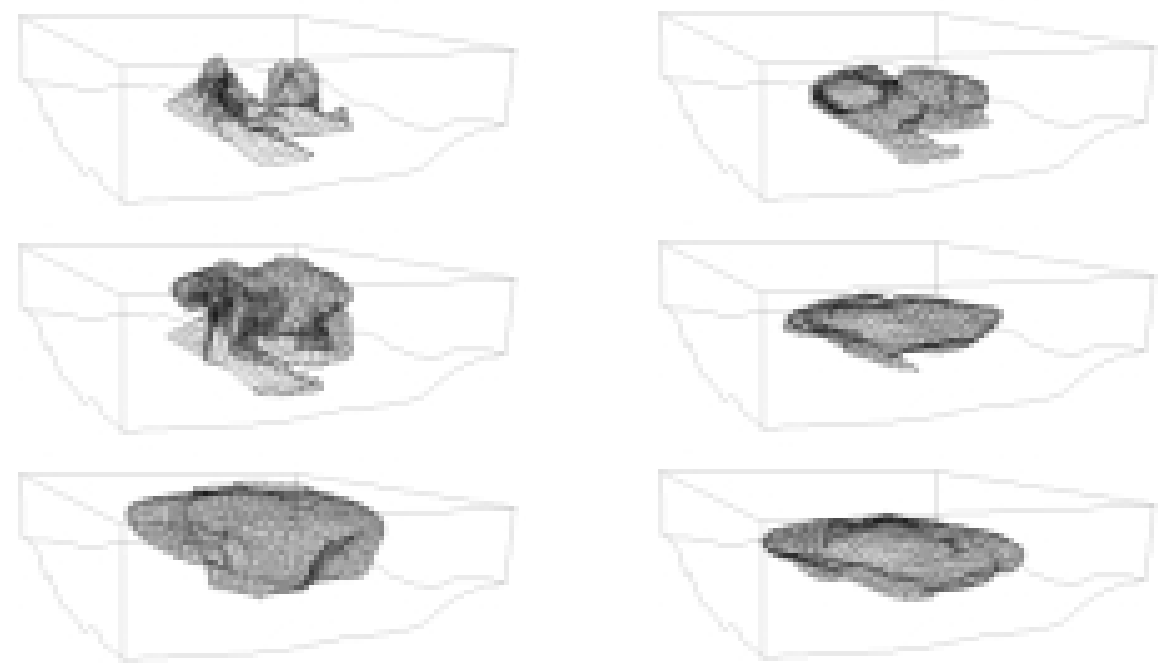

Fig. 8. Time evolution of concentration $(\langle C\rangle=0.01), \tau=1$ p.m., 6 p.m., day (I), 10 p.m., day (II) respectively; left- weak stratification, right- strong stratification.

reduces convective transport resulting in an increase of pollutant concentrations over residential and industrial zones.

\section{Conclusions}

Numerical simulations of combined effects of terrain orography and thermal stratification on pollutant dispersion in a town valley were performed using the time-dependent Reynolds-averaged Navier-Stokes method (T-RANS). The approach can be regarded as very large eddy simulation, with a single-point closure playing the role of the 'subscale' model. In comparison with the con- 
ventional LES, the model of the unresolved motion (here a reduced algebraic $\left\langle\theta u_{i}, c u_{i}, k-\varepsilon-\theta^{2}\right\rangle$ model) covers a much larger part of turbulence spectrum whereas the large deterministic structure was fully resolved. Validation of the proposed T-RANS approach was performed for situations where the effects of thermal stratification and terrain orography were separated, and for which a good experimental and numerical database exist. Unsteady turbulent penetrative convection of unstable mixed layer and convection over horizontal wavy walls demonstrated a very good predictive potential of the proposed approach. The full scale simulations of pollutant dispersion in a town valley with distinct residential and industrial zones under differently imposed thermal stratification, portrayed qualitatively very reasonable results and at the same time confirmed numerical efficiency and robustness of the proposed approach. We believe that the T-RANS can be used as a potentially powerful and efficient tool for prediction of local environments.

\section{References}

1. Deardorff, J. W. and Willis, G. E. and Lilly, D. K.: Laboratory investigation of non-steady penetrative convection. J. Fluid Mech., 35, (1969) 7-31,

2. Hunt, J.C.R.: UltraCFD for Computing Very Complex Flows. ERCOFTAC Bulletin, No.45, (2000) 22-23

3. Hanjalić, K. and Kenjereš, S.: T-RANS simulations of deterministic eddy structure in flows driven by thermal buoyancy and Lorentz force. Flow Turbulence and Combustion, Vol.66, (2001) 427-451

4. Kenjereš, S. and Hanjalić, K.: Transient analysis of Rayleigh-Bénard convection with a RANS model. Int. J. Heat and Fluid Flow, 20 (1999) 329-340

5. Krettenauer, K. and Schumann, U.: Numerical simulation of turbulent convection over wavy terrain. J. Fluid Mech., 237 (1992) 261-299

6. Mahrt, L.: Stratified Atmospheric Boundary Layers and Breakdown of Models. Theoret. Comput. Fluid Dynamics, 11 (1998) 263-279

7. Nieuwstadt, F. T. M. and Mason, P. J. and Moeng, C. H. and Schumann, U.: Largeeddy simulation of the convective boundary layer: A comparison of four computer codes. Proceedings of the 8th Turbulent Shear Flow Symposium, Munich, Germany (1991) 1.4.1-1.4.6

8. Schmidt, H. and Schumann, U.: Coherent structure of the convective boundary layer derived from large-eddy simulations. J. Fluid Mech., 200 (1989) 511-562

9. Stull, R. B.: An Introduction to Boundary Layer Meteorology. Kluwer Academic Publishers (1988)

10. Willis, G. E. and Deardorff, J. W.: Laboratory Model of Unstable Planetary Boundary Layer. J. Atmos. Sci., 31 (1974) 1297-1307 